\title{
Massive Pulmonary Thromboembolism in Pregnancy Rescued Using Transcatheter Thrombectomy
}

\author{
Tetsuya SATo, ${ }^{1} \mathrm{MD}$, Renpei KoBAtake, ${ }^{1} \mathrm{MD}$, Ryo YoshioKa, ${ }^{1} \mathrm{MD}$, \\ Soichiro FuKE, ${ }^{1} \mathrm{MD}$, Tetsuya IKEDA, ${ }^{1} \mathrm{MD}$, Hironori SAITO, ${ }^{1} \mathrm{MD}$, \\ Kiyoaki MAEKAwA, ${ }^{1} \mathrm{MD}$, and Toru HIOKA, ${ }^{1} \mathrm{MD}$
}

\begin{abstract}
SUMMARY
We report a rescued 37-year-old woman in her $30^{\text {th }}$ week of gestation with massive pulmonary thromboembolism who was admitted to our cardiac care unit with progressive dyspnea and 2 episodes of syncope. Helical chest CT showed massive pulmonary thromboembolism of both pulmonary arteries. Although 26,000 U/day of heparin was administered following insertion of a temporary filter, hemodynamic evaluation documented no improvement. Since pulmonary artery (PA) pressure increased from $62 / 22 \mathrm{mmHg}$ to 80 / $24 \mathrm{mmHg}$ just after an emergency cesarean section on day 2, an emergency transcatheter thrombectomy was performed and it showed decreased PA pressure following extensive thrombus aspiration. Mother and baby were discharged with no complications. (Int Heart J 2007; 48: 269-276)
\end{abstract}

Key words: Pregnancy, Pulmonary thromboembolism, Catheter thrombectomy, Rescued case

IN recent years, pulmonary thromboembolism (PTE) has surpassed infection, hemorrhage, and preeclampsia-eclampsia to become the leading cause of maternal mortality in the United States during pregnancy. ${ }^{1)}$ Furthermore, the incidence of PTE has been increasing in Asian countries, including Japan. ${ }^{2)}$ Although the treatment options available for patients with massive PTE in whom conservative heparin therapy has failed include thrombolytic therapy, catheter-directed thrombectomy, and surgical embolectomy, ${ }^{3)}$ there are no conclusive data or evidence on the appropriate treatment of massive PTE. Furthermore, to our knowledge, there are no case reports of pregnant patients rescued using emergency catheterdirected thrombectomy and the timing of cesarean section is controversial. We describe a 37-year-old pregnant woman with massive PTE rescued using catheter thrombectomy following an emergency cesarean section. The systemic condition of the patient was dramatically improved after the procedure and she and her

From the ${ }^{1}$ Department of Cardiology, Okayama Red Cross General Hospital, Okayama, Japan.

Address for correspondence: Tetsuya Sato, MD, Department of Cardiology, Okayama Red Cross General Hospital, 2-1-1 Aoe, Okayama City, Okayama 700-8607, Japan.

Received for publication November 17, 2006.

Revised and accepted January 22, 2007. 
baby were discharged in fairly good general condition.

\section{Case Report}

A 37-year-old woman, 30 weeks pregnant, with increasing dyspnea and 2 episodes of loss of consciousness lasting 1 minute, was admitted to our cardiac care unit. She had no history of thromboembolic disorder in her previous 2 deliveries. Physical examination revealed blood pressure of $108 / 80 \mathrm{mmHg}$, heart rate of $114 / \mathrm{min}, 88 \%$ oxygen saturation on room air, a BMI of 21.4 , grade III/VI systolic ejection murmur at the upper left sternal border, no varicosis of the lower extremities, and mild edema of the left lower extremity. Laboratory findings on admission showed a D-dimer level of $3.7 \mu \mathrm{g} / \mathrm{mL}$, WBC of 11600 , AST of $25 \mathrm{IU} /$ L, ALT of 19 IU/L, LDH of $208 \mathrm{IU} / \mathrm{L}$, and normal ranges for lupus anticoagulant, anticardiolipin antibody titer, and protein $\mathrm{S}$ and $\mathrm{C}$ activities. Arterial blood gas on $3 \mathrm{~L}$ of $100 \%$ oxygen by nasal cannula showed alkalosis ( $\mathrm{pH} 7.47$ ), carbon dioxide pressure $\left(\mathrm{PCO}_{2}\right)$ of $25 \mathrm{mmHg}$, and oxygen pressure $\left(\mathrm{PO}_{2}\right)$ of $69 \mathrm{mmHg}$. Electrocardiography showed sinus rhythm, right axis deviation, $\mathrm{S}$ wave in lead I, and Q and inverted $\mathrm{T}$ waves in lead III. Transthoracic echocardiography demonstrated overload of the right ventricle (right ventricular end-diastolic diameter of 42 $\mathrm{mm}$ ). Helical chest CT with lead shields revealed massive thromboembolism in both pulmonary arteries (Figure 1).

The patient underwent emergency insertion of a temporary inferior vena cava filter (Neuhaus Protect, Toray Medical, Tokyo) through the right internal
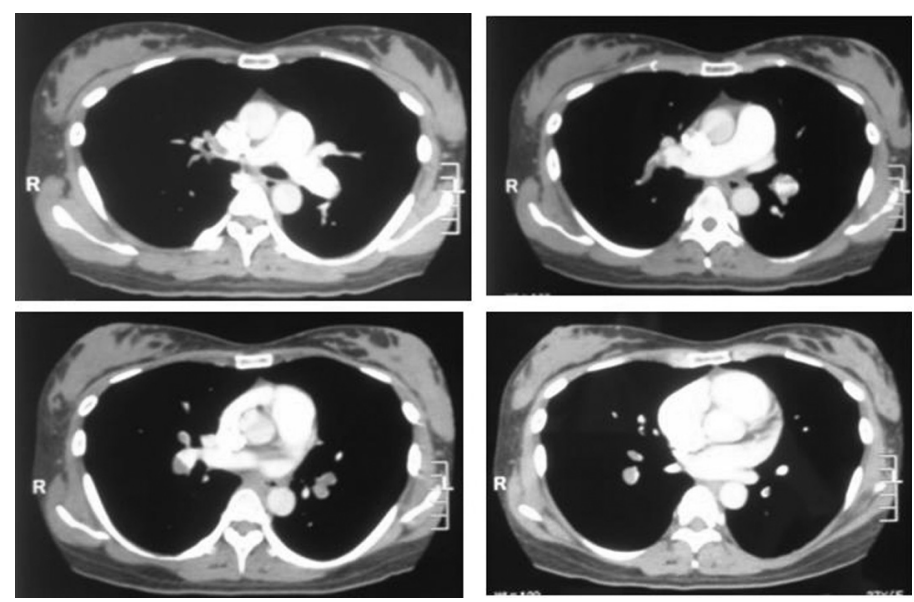

Figure 1. Helical chest $\mathrm{CT}$ images on admission.

Note the massive thromboembolism in the right superior, medial, and inferior lobe pulmonary artery and in the left basal pulmonary artery. 
jugular vein. Deployment was checked by fluoroscopy, which was performed quickly, with lead shields protecting the fetus from exposure to radiation. The patient wore an elastic stocking on both lower extremities. Although 26,000 U/ day of heparin, maintaining the activated partial thromboplastin time from 70 to 80 seconds, was administered intravenously, even with catecholamine, her symptoms became worse and her hemodynamic evaluation documented no improvement in PA pressure of 62/22 mmHg, hyperglycemia, and blood pressure of 100/ $60 \mathrm{mmHg}$ (Figure 2). Treatment options were discussed because of her worsening condition and the perceived high risk of a fatal PTE. A decision was made to

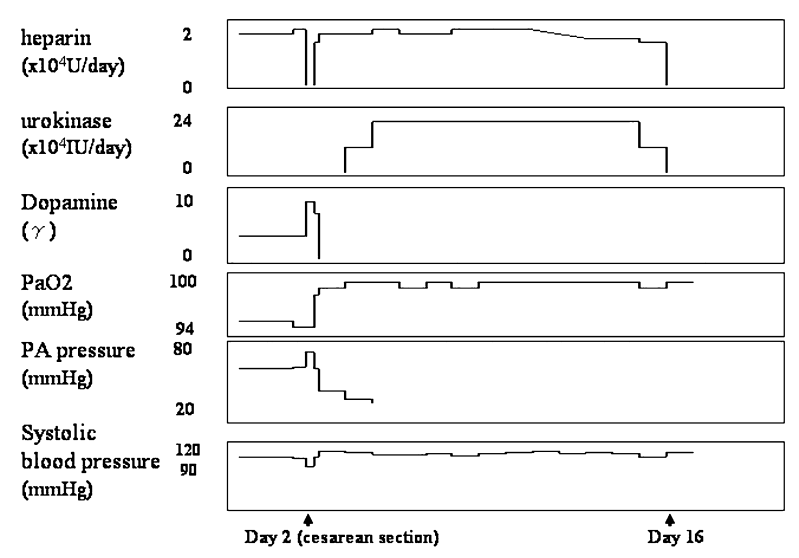

Figure 2. Clinical course after admission.

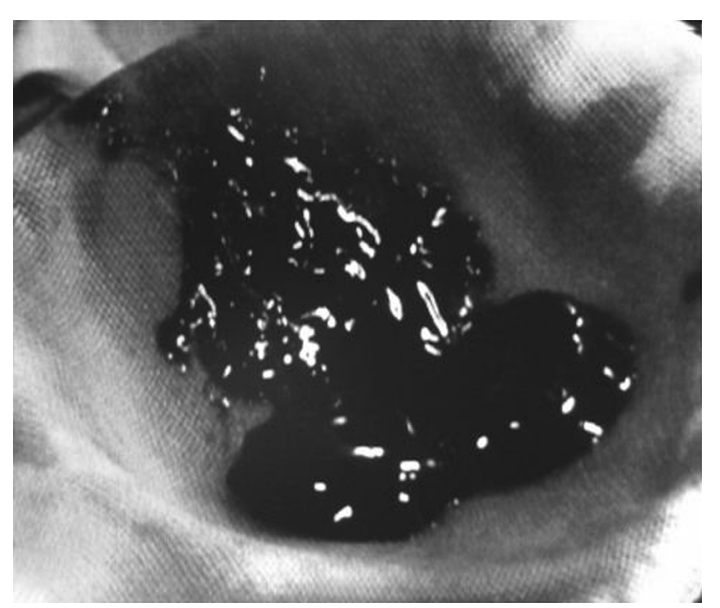

Figure 3. Aspirated thrombus.

Note the large amount of thrombus that was aspirated. 
attempt an emergent cesarean section under general anesthesia on day 2 to stop the hypercoagulability condition induced by pregnancy and to start more effective thrombolysis therapy.

Just after the operation, her PA pressure increased from $62 / 22 \mathrm{mmHg}$ to 80 / $24 \mathrm{mmHg}$ and blood pressure decreased from $100 / 60 \mathrm{mmHg}$ to $90 / 58 \mathrm{mmHg}$ (Figure 2), indicating that more thrombus would be passing through the filter. Emergent transcatheter thrombectomy using 8F JR-4.0 (Johnson \& Johnson-Cordis, Miami Lakes, USA) and 7F Multipurpose (Medtronic Inc., Minneapolis, USA) catheters through the left internal jugular vein was performed following $5000 \mathrm{U}$ of heparin administered intravenously. A significant thrombus was aspirated (Figure 3). Systolic PA pressure decreased from $80 \mathrm{mmHg}$ to $60 \mathrm{mmHg}$ and systolic blood pressure increased from $90 \mathrm{mmHg}$ to $106 \mathrm{mmHg}$, showing a dramatic recovery just after this procedure. Left pulmonary angiography showed a significantly decreased amount of thrombus (Figure 4) and recovery of blood flow.

Postoperatively, anticoagulant was maintained with the continuous intravenous administration of heparin at a dosage to maintain the activated partial thromboplastin time at 1.5 to 2 times that of the control value. Intravenous administration of urokinase at $12 \times 10^{4} \mathrm{IU} /$ day was started on day 3 , increased up to $24 \times 10^{4} \mathrm{IU} /$ day on day 4 , and continued for 2 weeks (Figure 2). Systolic PA pressure decreased to $40 \mathrm{mmHg}$ on day 3 and $28 \mathrm{mmHg}$ on day 4. Pelvic CT showed the thrombus in the left femoral vein on day 6 but it had disappeared on day 14 (Figure 5). There were no bleeding complications during thrombolytic therapy. The patient was discharged the following day without complications.

A

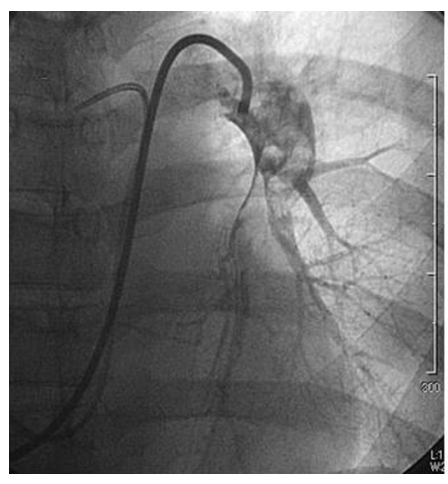

B

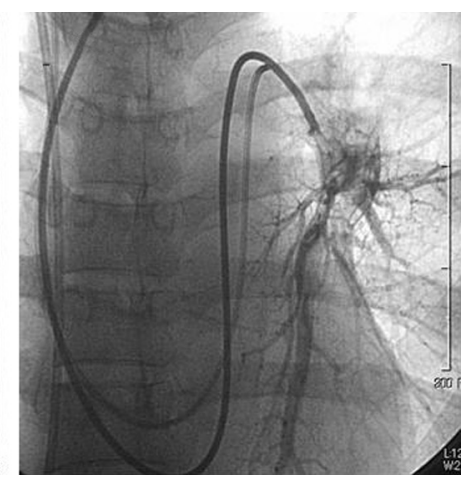

Figure 4. Pulmonary arteriography precatheter (A) and postcatheter thrombectomy (B). A: Large amount of thrombus (filling defect) can be observed in the left basal pulmonary artery.

B: Significant decrease in size of thrombus can be seen. 


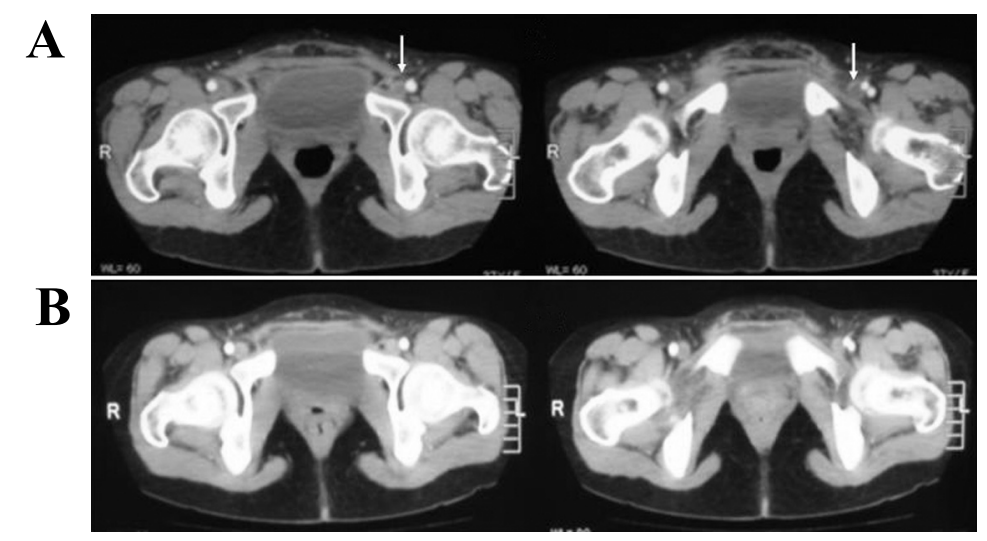

Figure 5. Pelvic CT images on day 6 (A) and 14 (B). A: Note the thrombus in the left femoral vein on day 6 B: Thrombus had disappeared on day 14 .

\section{DISCUSSION}

This case highlights the importance of emergent transcatheter thrombectomy for pregnant patients with severely unfavorable systemic conditions induced by PTE. The most important point in this case was the timing of the cesarean section. This depends on the gestation week, effect of thrombolytic therapy, hemodynamics, and systemic condition of the mother.

Unfortunately, heparin was not sufficiently effective in this patient. Heparin, a large molecule, is the mainstay of therapy for PTE during pregnancy ${ }^{4}$ because it does not cross the placenta, ${ }^{5)}$ and does not carry risks of fetal hemorrhage or teratogenesis. Although its use during pregnancy seems to be safe, the dosing remains controversial. The dose requirements may be as high as twice the normal weight-based dose because of increased heparin degradation by the placenta. ${ }^{6}$ Although about twice the usual dose of heparin was also administered in this case, heparin therapy may be insufficient to manage the patient even with the therapeutic dose in massive PTE as in this case. Supportive care and other anticoagulation should be instituted without delay. Subsequent treatment options are controversial and include thrombolytics and embolectomy. ${ }^{7)}$

Urokinase is a small molecule purified from human urine that can cross the placenta. ${ }^{3)}$ Although there are some case reports of pregnant patients receiving urokinase for PTE with a favorable clinical outcome, ${ }^{8)}$ there is substantially less reported experience because of concerns about bleeding, teratogenicity, and abruptio placentae praematurus. Furthermore, there are case reports and case 
studies in the thrombolysis literature of recombinant tissue plasminogen activator or streptokinase being used safely in pregnancy. ${ }^{3)}$ However, there are no prospective randomized trials to date that have evaluated the efficacy and safety of thrombolytics during pregnancy, and these studies are unlikely to be undertaken in the future. Furthermore, our obstetricians were concerned about major bleeding as a complication of thrombolysis just after the operation. Therefore, thrombolytic treatment was not performed in this case.

A normal pregnancy itself induces a hypercoagulable state that is multifactorial and is thought to be due to a combination of physical and hormonal factors as well as hematologic changes. ${ }^{4)}$ Some mechanisms are also explained with Virchow's classic triad of factors underlying venous thrombosis; hypercoagulability, venous stasis, and vascular damage. ${ }^{9)}$ We therefore considered that resolving the pregnancy as soon as possible would be very important for improving the hypercoagulability stage and for recovery of her general condition.

The incidence of fatal PTE is approximately 10 times greater after a cesarean delivery than after a vaginal delivery. ${ }^{10)}$ Furthermore, the incidence of PTE following a cesarean is twice as high as that following gynecologic surgery, ${ }^{2}$ indicating that cesarean section itself produces an environment that results in PTE. However, this woman was in the $30^{\text {th }}$ week of gestation and it was difficult to perform a vaginal delivery. The life of the mother was threatened even with heparin administration, indicating that conventional therapy had failed. We therefore decided to perform an emergent cesarean section on day 2 .

Just after the operation, systolic PA pressure increased to almost the same degree as systolic blood pressure, indicating hemodynamic compromise and more thrombus passing through the filter, although the thrombus may have shifted in the pulmonary artery. Aburahma, et $a l^{11)}$ reported no serious maternal or fetal complications in pregnant women with permanent filters inserted for the management of deep vein thrombosis (DVT). However, $27 \%$ had significant lower extremity edema at a mean of 61 months follow-up. Because of long-term safety concerns, an attractive alternative to permanent filters is to place a temporary filter. ${ }^{12)}$ A temporary filter was inserted on an emergency basis because the risk of recurrent PTE was high and concern about complications of residual DVT. The indications for insertion of a temporary filter at our hospital are; 1) high risk of recurrent PTE, such as patients with residual DVT who have a transient risk factor, ${ }^{13)}$ 2) severe overload of the right-side of the heart, 3) floating thrombus, and 4) high risk of bleeding induced by thrombolysis therapy.

However, the capture rate by different temporary filters of thrombi $3 \mathrm{~mm}$ or $5 \mathrm{~mm}$ in diameter in a $21 \mathrm{~mm}$ diameter vena cava model ranged from $44 \%$ to $94 \%$ and $60 \%$ to $96 \%$, respectively. ${ }^{14)}$ Even small thrombi may cause symptoms and even death if they reach a previously compromised lung. ${ }^{15)}$ Therefore, we should 
pay attention to PTE recurrence in women undergoing a cesarean section even with a vena cava filter. Furthermore, recurrent PTE might indicate amniotic fluid embolism during a cesarean section.

An alternative technique in patients with massive PTE in whom conventional thrombolysis therapy has failed is catheter embolectomy employing a large steerable catheter with a suction cup on the end. ${ }^{16)}$ The results of the only 2 studies published showed that embolus extraction is achieved in about two-thirds of patients and mortality in these studies was about $30 \% .{ }^{17)}$ Although there are a few studies ${ }^{18,19)}$ describing the clinical benefits of thrombectomy using other kinds of catheters, this procedure was rarely undertaken in pregnant patients in the literature. It was performed quickly and safely and the hemodynamics recovered dramatically. We recommend catheter thrombectomy as an important alternative treatment strategy in pregnant patients with massive PTE.

PTE, which has increased in recent years, is 4 to 6 times more common during pregnancy compared with the general population ${ }^{20)}$ and is a major cause of maternal mortality and the third highest cause of maternal death in Japan. ${ }^{21)}$ Risk factors for PTE in the antenatal period include a maternal age exceeding 35 years, a parity of 3 or more, body weight greater than $80 \mathrm{~kg}$, a personal or family history of PTE, varicose veins, smoking, and known antiphospholipid syndrome. ${ }^{22,23)}$ The risks in this case were only age and parity number. Therefore, we should pay attention to the chance of PTE for every pregnant woman, even if she is considered to be at low risk.

\section{REFERENCES}

1. Callaghan WM, Berg CJ. Pregnancy-related mortality among women aged 35 years and older, United States, 1991-1997. Obstet Gynecol 2003; 102: 1015-21.

2. Chisaka H, Utsunomiya H, Okamura K, Yaegashi N. Pulmonary thromboembolism following gynecologic surgery and cesarean section. Int J Gynaecol Obstet 2004; 84: 47-53.

3. Ahearn GS, Hadjiliadis D, Govert JA, Tapson VF. Massive pulmonary embolism during pregnancy successfully treated with recombinant tissue plasminogen activator: a case report and review of treatment options. Arch Intern Med 2002; 162: 1221-7.

4. Stone SE, Morris TA. Pulmonary embolism during and after pregnancy. Crit Care Med 2005; 33: S294-300. (Review)

5. Ginsberg JS, Hirsh J, Turner DC, Levine MN, Burrows R. Risks to the fetus of anticoagulant therapy during pregnancy. Thromb Haemost 1989; 61: 197-203. (Review)

6. Barbour LA, Smith JM, Marlar RA. Heparin levels to guide thromboembolism prophylaxis during pregnancy. Am J Obstet Gynecol 1995; 173: 1869-73.

7. Stone SE, Morris TA. Pulmonary embolism and pregnancy. Crit Care Clin 2004; 20: 661-77. (Review)

8. Kramer WB, Belfort M, Saade GR, Surani S, Moise KJ Jr. Successful urokinase treatment of massive pulmonary embolism in pregnancy. Obstet Gynecol 1995; 86: 660-2.

9. Leonhardt G, Gaul C, Nietsch HH, Buerke M, Schleussner E. Thrombolytic therapy in pregnancy. J Thromb Thrombolysis 2006; 21: 271-6. (Review) 
10. Bonnar J. Can more be done in obstetrics and gynecologic practice to reduce morbidity and mortality associated with venous thromboembolism? Am J Obstet Gynecol 1999; 180: 784-91.

11. Aburahma AF, Boland JP. Management of deep vein thrombosis of the lower extremity in pregnancy: a challenging dilemma. Am Surg 1999; 65: 164-7.

12. Ishikawa $\mathrm{T}$, Okabe $\mathrm{T}$, Ogawa $\mathrm{K}$, et al. Usefulness of a second temporary vena cava filter for preventing acute pulmonary thromboembolism. Circ J 2003; 67: 718-20.

13. Kai R, Imamura H, Kumazaki S, et al. Temporary inferior vena cava filter for deep vein thrombosis and acute pulmonary thromboembolism: effectiveness and indication. Heart Vessels 2006; 21: 221-5.

14. Lorch H, Zwaan M, Kulke C, Weiss HD. In vitro studies of temporary vena cava filters. Cardiovasc Intervent Radiol 1998; 21: 146-50.

15. Hsu JT, Chu CM, Chang ST, Cheng HW, Cheng NJ, Chung CM. Prognostic role of right ventricular dilatation and troponin I elevation in acute pulmonary embolism. Int Heart J 2006; 47: 775-81.

16. Riedel M. Acute pulmonary embolism 2: treatment. Heart 2001; 85: 351-60. (Review)

17. Riedel M. Therapy of pulmonary thromboembolism. Part I: acute massive pulmonary embolism. Cor Vasa 1996; 38: 93-102.

18. Yoshida M, Inoue I, Kawagoe T, et al. Novel percutaneous catheter thrombectomy in acute massive pulmonary embolism: rotational bidirectional thrombectomy (ROBOT). Catheter Cardiovac Interv 2006; 68: 112-7.

19. Schmitz-Rode T, Janssens U, Duda SH, Erley CM, Gumther RW. Massive pulmonary embolism: percutaneous emergency treatment by pigtail rotation catheter. J Am Coll Cardiol 2000; 36: 375-80.

20. Greer IA, Thomson AJ. Management of venous thromboembolism in pregnancy. Best Pract Res Clin Obstet Gynaecol 2001; 15: 583-603. (Review)

21. Statistics and Information Department, Minister's Secretariat. Trends in maternal death and maternal mortality rates (per 100,000 total births) by cause of death: Vital Statistics of Japan 2002 Vol. 1. Ministry of Health, Labour and Welfare 2004; 316, Japan.

22. Refuerzo JS, Hechtman JL, Redman ME, Whitty JE. Venous thromboembolism during pregnancy. Clinical suspicion warrants evaluation. J Reprod Med 2003; 48: 767-70.

23. Chen JY, Chao TH, Guo YL, et al. A simplified clinical model to predict pulmonary embolism in patients with acute dyspnea. Int Heart J 2006; 47: 259-71. 